\title{
Automatic Fontanel Extraction from Newborns' CT Images Using Variational Level Set
}

\author{
Kamran Kazemi $^{1,2}$, Sona Ghadimi ${ }^{2,3}$, Alireza Lyaghat ${ }^{1}$, Alla Tarighati ${ }^{1}$, \\ Narjes Golshaeyan ${ }^{1}$, Hamid Abrishami-Moghaddam ${ }^{2,3}$, Reinhard Grebe ${ }^{2}$, \\ Catherine Gondary-Jouet ${ }^{4}$, and Fabrice Wallois ${ }^{2,5}$ \\ ${ }^{1}$ Shiraz University of Technology, Shiraz, Iran \\ ${ }^{2}$ GRAMFC EA 4293, Faculté de Médecine, Université de Picardie Jules Verne, \\ Amiens, 80036, France \\ ${ }^{3}$ K.N. Toosi University of Technology, Tehran, Iran \\ ${ }^{4}$ Department of Neuroradiology, Centre Hospitalier Universitaire d'Amiens, \\ 80054, Amiens, France \\ ${ }^{5}$ GRAMFC, EFSN Pediatrique, Centre Hospitalier Universitaire d'Amiens, \\ 80054, Amiens, France \\ \{kamran.kazemi, reinhard.grebe, fabrice wallois, \\ hamid.abrishami\}u-picardie.fr, \\ gondry-jouet.catherine@chu-amiens.fr, sona_ghadimi@ee.kntu.ac.ir
}

\begin{abstract}
A realistic head model is needed for source localization methods used for the study of epilepsy in neonates applying Electroencephalographic (EEG) measurements from the scalp. The earliest models consider the head as a series of concentric spheres, each layer corresponding to a different tissue whose conductivity is assumed to be homogeneous. The results of the source reconstruction depend highly on the electric conductivities of the tissues forming the head.The most used model is constituted of three layers (scalp, skull, and intracranial). Most of the major bones of the neonates' skull are ossified at birth but can slightly move relative to each other. This is due to the sutures, fibrous membranes that at this stage of development connect the already ossified flat bones of the neurocranium. These weak parts of the neurocranium are called fontanels. Thus it is important to enter the exact geometry of fontaneles and flat bone in a source reconstruction because they show pronounced in conductivity. Computer Tomography (CT) imaging provides an excellent tool for non-invasive investigation of the skull which expresses itself in high contrast to all other tissues while the fontanels only can be identified as absence of bone, gaps in the skull formed by flat bone. Therefore, the aim of this paper is to extract the fontanels from CT images applying a variational level set method. We applied the proposed method to CT-images of five different subjects. The automatically extracted fontanels show good agreement with the manually extracted ones.
\end{abstract}

Keywords: newborns, fontanel, source reconstruction, level set, segmentation.

\section{Introduction}

The electroencephalogram (EEG) measures ongoing electrical activity of the brain recorded from electrodes placed on the scalp. It is widely used in clinical setting for the 
diagnosis and management of epilepsy in adults and children. EEG provides the necessary data for non-invasive estimation of the location of electrical sources in the brain. For these estimates inverse methods are used where location, amplitude, and orientation of a dipole source is adjusted to a model of the head to obtain the best fit between the measured EEG's and those produced by the source in the model [1-3]. In addition to other factors such as noise, errors of location measurement, etc., the accuracy of these estimates depends highly on the accuracy of the head model [4]. Magnetic resonance imaging (MRI) and computed tomography (CT) are the two major neuroimaging modalities that can be used for multi-component head model construction.

While MRI provides an excellent tool for non-invasive study of brain tissues it is not suitable for accurate skull extraction especially in newborns which have a very thin skull in comparison to adults. CT scan uses X-ray beams passing through the sample at a series of different angles. Based on the attenuation of these beams, an image can be constructed. Due to high its attenuation, the bone appears bright in CT images. Therefore, CT provides an excellent non-invasive tool for skull study and modeling.

Most of the major bones of the neonates' skull are ossified at birth but can slightly move relative to each other. This is due to the sutures, fibrous membranes that at this stage of development connect the already ossified flat bones of the neurocranium. These weak parts of the neurocranium are called fontanels [5]. Thus, fontanels are the narrow seams of fibrous connective tissue that separate the flat bones of the skull. They can be identified as gaps between two cranial bones in newborns' CT images. Newborns have six fontanels: the anterior and the posterior, two mastoid, and two sphenoid ones [5]. These fontanels have different electrical properties, conductivities, in comparison to the cortical bone. This difference may have not neglectable influence on the localization of the electrical sources in the brain [6]. Thus, accurate modeling of the fontanels will improve the results of source localization for newborns' brain.

The present study aimed to extract the cortical bone and fontanels from newborns' CT images. The extracted tissues then can be combined with the extracted brain tissues from MRI to construct a complete realistic 3D head model for newborns.

The rest of the paper is organized as follows. Section 2 presents our method of cortical bone and fontanel extraction from CT images. The extracted fontanels and evaluation results are presented in section 3. Finally concluding remarks and discussion are given in section 4 .

\section{Materials and Methods}

Figure 1 shows the block diagram of our method for bone and fontanel extraction from newborns' CT images. As shown, the cortical bone is extracted from CT images applying an automatic thresholding method. Then, the level set method of minimal surface reconstruction as proposed by Zhao et al., [7] is used to determine the inner and outer surface of a closed skull model based on the before extracted cortical bone. Finally, the fontanels can be extracted by removing the bone from the constructed closed skull model. 


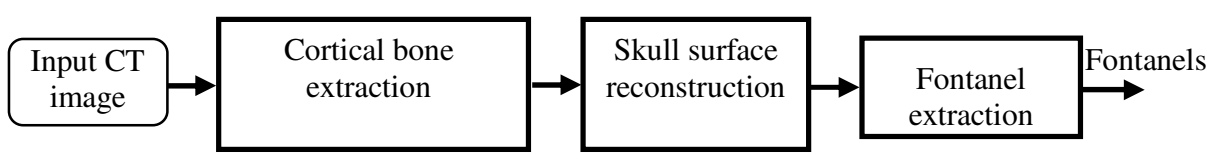

Fig. 1. Cortical bone and fontanel extraction method

\subsection{Data Acquisition}

The developed method has been applied to the 3D volumetric CT images of five newborns aged between 40 and 42 weeks at the date of examination (gestational age). The CT images have axial orientation with data matrix of $256 \times 256$ pixels and voxel size $0.75 \times 0.75 \times 1.25 \mathrm{~mm}^{3}$.

\subsection{Cortical Bone Extraction}

Due to the high contrast between bone and adjacent tissues in CT images of the head, cortical bones can be extracted by simple automatic histogram thresholding [8]. The extracted bones are labeled $V_{\text {bone }}$ and used to construct the inner and outer skull surface.

\subsection{Skull Surface Reconstruction}

In order to reconstruct the inner and outer skull (bone and fontanel) surfaces from extracted cortical bones, the variational level set method and tagging algorithm proposed by Zhao et al. [7] are applied. The method originally developed for surface reconstruction from unorganized data.

Minimum surface-like model. Let $\mathrm{S}$ denote the data set which includes the before determined cortical bone surface patches. So, the basic problem is determining an $\mathrm{N}$ dimensional surface $\Gamma$ which minimizes the following energy functional:

$$
E(\Gamma)=\left[\int_{\Gamma} d^{p}(x) d s\right]^{\frac{1}{p}}, \quad 1 \leq p \leq \infty
$$

where $d(\mathbf{x})$ is the distance function $(\operatorname{dist}(\mathbf{x}, S))$ from the point $\mathbf{x}$ to $S, \Gamma$ is an arbitrary surface and $d s$ is the surface area. Assuming $\Gamma(\mathrm{t})$ to be the zero level set of a function $\phi(x, t)$ :

$$
\Gamma(t)=\{x: \phi(x, t)=0\}
$$

and satisfying the following conditions:

$$
\begin{array}{ll}
\phi(x, t)<0 & \text { for } x \in \Omega \\
\phi(x, t)=0 & \text { for } x \in \partial \Omega=\Gamma(t) \\
\phi(x, t)>0 & \text { for } x \in \bar{\Omega}
\end{array}
$$

where $\Omega(t)$ is the region enclosed by $\Gamma(t)$. 
As derived in [7], the variational level set formulation for energy functional (1) is:

$$
E(\Gamma)=\left[\int_{\Gamma} d^{p}(x) d s\right]^{1 / p}=E(\phi)=\left[\int d^{p}(x) \delta(\phi(x))|\nabla \phi(x)| d x\right]^{1 / p}
$$

where $\delta(x)$ is the one-dimensional delta function and $\delta(\varphi(\mathbf{x}))|\nabla \varphi(\mathbf{x})| d \mathbf{x}$ is the surface area element at the zero level set of $\varphi$. With the corresponding gradient flow for $\varphi$ and extending the motion to all level sets by replacing $\delta(\varphi)$ with $|\nabla \varphi|$, the level set formulation for gradient flow will obtain as follow:

$$
\frac{\partial \phi}{\partial t}=\frac{1}{p}|\nabla \phi|\left[\int d^{p}(x) \delta(\phi)|\nabla \phi| d x\right]^{1 / p^{-1}} \nabla \cdot\left[d^{p}(x) \frac{\nabla \phi}{|\nabla \phi|}\right]
$$

Though this level set can be used to smooth the implicit surface. For more and fast post smoothing, the level set proposed by Whitaker [9] is used using the variational level set formulation. Let $\varphi_{0}$ denote the initial level set function whose zero level set is the surface to be smoothed. The smoothed implicit surface is defined as the zero level set of $\varphi$ that minimizes the following functional:

$$
\frac{1}{2} \int\left(H(\phi)-H\left(\phi_{0}\right)\right)^{2} d x+\varepsilon \int \delta(\phi)|\nabla \phi| d x,
$$

where $H(\mathbf{x})$ is the one dimensional Heaviside function. The first term in the above energy functional is a fidelity term that measures the symmetric volume difference between two closed surfaces. The second integral in the above functional is the surface area of the zero level set of $\phi$, which is a regularization term that minimizes the surface area of the smoothed surface. The constant $\varepsilon$ is a parameter that controls the balance between the fidelity and the regularization. We again find the minimizer by following the gradient flow of (6), whose level set formulation is:

$$
\frac{\partial \phi}{\partial t}=|\nabla \phi|\left[\varepsilon \kappa-\left(H(\phi)-H\left(\phi_{0}\right)\right)\right]
$$

Initial surface. The surface reconstruction can be started with a simple initial surface such as cube or a sphere. However, if the initial surface is too far from the real shape, the PDE evolution takes a long time and computational cost. Therefore, a good initial surface helps to speed up convergence to the equilibrium surface. On a rectangular grid, an implicit surface can be used as an interface that separates the exterior grids from the interior grids.

The tagging algorithm proposed by Zhao [10] aims to identify as many correct exterior grids as possible and hence provides a good initial implicit surface. Since the size of the images in our application is rather big, applying this algorithm becomes very time consuming, therefore, we introduce here our modified tagging algorithm. We start from any initial exterior region that is a subset of the true exterior region. All grids that are not in the initial exterior region are labeled as interior grids. Those interior grids that have at least one exterior neighbor are labeled as temporary boundary grids. Now to march the temporary boundary, the Euclidean distance between all 
grids and the extracted newborns' cortical bones is computed. Then all temporary boundary voxels which are not on the data sets are checked one by one. If it has an interior neighbor with a large or equal distance, this temporary boundary point will be considered as a final boundary point. Otherwise, this temporary boundary point will be returned into an exterior point.

Reconstruction of inner and outer skull surface. The described level set method is applied to reconstruct the inner and outer skull surfaces $\left(\Gamma_{\text {in }}\right.$ and $\Gamma_{\text {out }}$ respectively) based on the extracted cortical bones. The inner surface $\left(\Gamma_{i n}\right)$ is extracted by initializing the zero level set of function $\phi_{i n}(x, t)$ inside the skull. According to equation (3), $\phi_{i n}(x, t)$ is negative inside $\Gamma_{i n}$ and positive outside $\Gamma_{i n}$. Thus a negative value for $\phi_{i n}(x, t)$ indicates the intracranial volume which is named $V_{I C}$. Accordingly, the outer skull surface $\Gamma_{\text {out }}$ is extracted by initializing the zero level set of function $\phi_{\text {out }}(x, t)$ outside the skull. Here a negative value of $\phi_{\text {out }}(x, t)$ indicates the intracranial (IC) and skull volume $\left(V_{I C \text {-skull }}\right)$.

\subsection{Fontanel Extraction}

In order to extract the fontanels, the obtained $V_{I C}$ and $V_{\text {bone }}$ are combined and a morphological filling filter is applied to fill eventual holes $\left(V_{I C \text {-bone }}\right)$. Then, by removing $V_{I C-b o n e}$ from $V_{I C-s k u l l}$ the fontanels are extracted $\left(V_{\text {fontanel }}\right)$.

$$
\begin{aligned}
& V_{\text {IC-bone }}=\operatorname{fill}\left(V_{I C} \mid V_{\text {bone }}\right) \\
& V_{\text {fontanel }}=\operatorname{XOR}\left(V_{\text {IC-bone }}, V_{I C-\text { skull }}\right)
\end{aligned}
$$

\subsection{Evaluation}

Quantitative evaluation of the results has been performed by calculating the similarity between the automatically extracted fontanels $\left(L_{a}\right)$ and the corresponding manually extracted ones (ground truth, $L_{m}$ ). The results were evaluated using the following similarity index (SI):

$$
S I=\frac{2 \mathrm{n}\left(L_{a} \cap L_{m}\right)}{n\left(L_{a}\right)+n\left(L_{m}\right)}
$$

\section{Results}

The obtained results demonstrate that the developed method reliably extracts fontanels from newborns' CT images. Figure 2 shows the CT image of a selected subject, its extracted cortical bone, inner and outer skull surfaces and finally the extracted fontanel. As can be seen in Figures 2.c and 2.e, the inner and outer surfaces are closed surfaces. The fontanels are closing smoothly the gaps left by the before detected flat bones, both forming together the closed skull model. Therefore, by removing these bones from the closed skull, the fontanels can be obtained (Figure 2.h). 

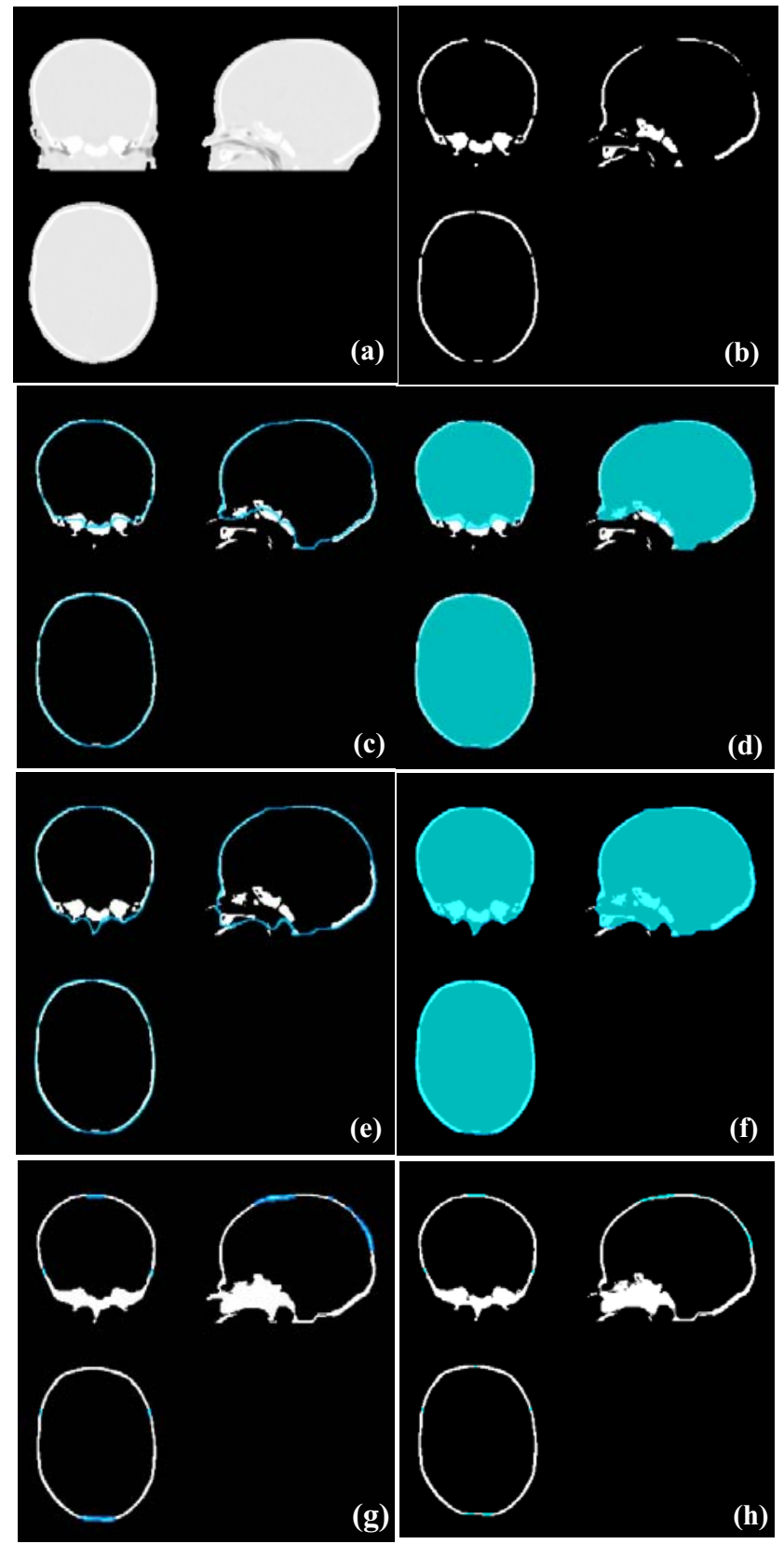

Fig. 2. Fontanel extraction. (a) Input $\mathrm{CT}$ image, (b) cortical bone after automatic thresholding, (c) final inner skull surface, (d) $\mathrm{V}_{\text {IC }}$, (e) final outer skull surface, (f) $\mathrm{V}_{\text {IC-skull }}$, (g) skull volume including extracted fontanels and bone, (h) manually extracted fontanels. 
Table 1 shows the quantitative results for three subjects obtained according to the similarity index. The results show $76 \pm 4 \%$ similarity for the automatically extracted fontanels with the corresponding ground truth.

Table 1. Similarity index, providing a measure for the similarity between automatically and manually extracted fontanels from newborns' CT images applying variational level set

\begin{tabular}{c|c}
\hline & Similarity Index \\
\hline Subject 1 & 0.74 \\
\hline Subject 2 & 0.75 \\
\hline Subject 3 & 0.72 \\
\hline Subject 4 & 0.80 \\
\hline Subject 5 & 0.82 \\
\hline
\end{tabular}

\section{Discussion and Conclusion}

In this paper we presented our approach for extracting fontanels from newborns' CT images based on variational level set method. Quantitative and qualitative results demonstrate the accuracy of the developed method for fontanel extraction from newborns.

The extracted fontanels and cortical bone in conjunction with the brain tissue models (after coregistration) may provide a realistic head model that can be used for applications such as source localization in newborns. This is important because of the different electrical conductivity of fontanels with respect to bone. In addition, the automatically extracted fontanels can be used for determining growth patterns for the newborns' skull.

\section{Acknowledgment}

This work was partially supported by the Center for International Research \& Collaboration of Iran (ISMO) under the grant number 86/13 and EGIDE France under the grant number 18639PL (Jundi Shapour scientific collaboration program).

\section{References}

1. Mosher, J.C., Leahy, R.M.: Recursive MUSIC: A Framework for EEG and MEG Source Localization. IEEE Trans. on Biomedical Engineering 45(11) (November 1998)

2. Liu, H., Gao, X., Schimpf, P.H., Yang, F., Gao, S.: A Recursive Algorithm for the ThreeDimensional Imaging of Brain Electric Activity: Shrinking LORETA-FOCUSS. IEEE Trans. on Biomedical Engineering 51(10) (October 2004)

3. Baillet, S., Riere, J.J., Marin, G., Mangin, J.F., Aubert, J., Garnero, L.: Evaluation of inverse methods and head models foe EEG source localization using a human skull phantom. Physics in Medicine and Biology 46(1), 77-96 (2001) 
4. Cuffin, B.N.: EEG Localization Accuracy Improvements Using Realistically Shaped Head Models. IEEE Trans. on Biomedical Imaging 43(3) (March 1996)

5. Kiesler, J., Richer, R.: The Abnormal Fontanel. American Family Physician 67(12) (June 2003)

6. Roche-Labarbe, N., Aarabi, A., Kongolo, G., Gondry-Jouet, C., Dümpelmann, M., Grebe, R., Wallois, F.: High-resolution EEG and source localization in neonates. Human Brain Mapping 29(2), 167-176 (2008)

7. Zhao, H.K., Osher, S., Merriman, B., Kang, M.: Implicit and non-parametric shape reconstruction from unorganized points using variational level set method. Computer Vision and Image Understanding 80(3), 295-319 (2000)

8. Otsu, N.: A threshold selection method from gray-level histograms. IEEE Trans. Sys. Man. Cyber. 9, 62-66 (1979)

9. Whitaker., R.: A level set approach to 3D reconstruction from range data. International Journal of Computer Vision (1997)

10. Zhao, H., Osher, S.: Contributed chapter. In: Osher, S., Paragios, N. (eds.) Geometric Level Set Methods in Imaging, Vision and Graphics. Springer, Heidelberg (2002) 